\title{
Esame di Stato: mai più senza prove scritte
}

\section{Paolo D'Achille}

PUBBLICATO: 13 SETTEMBRE 2021

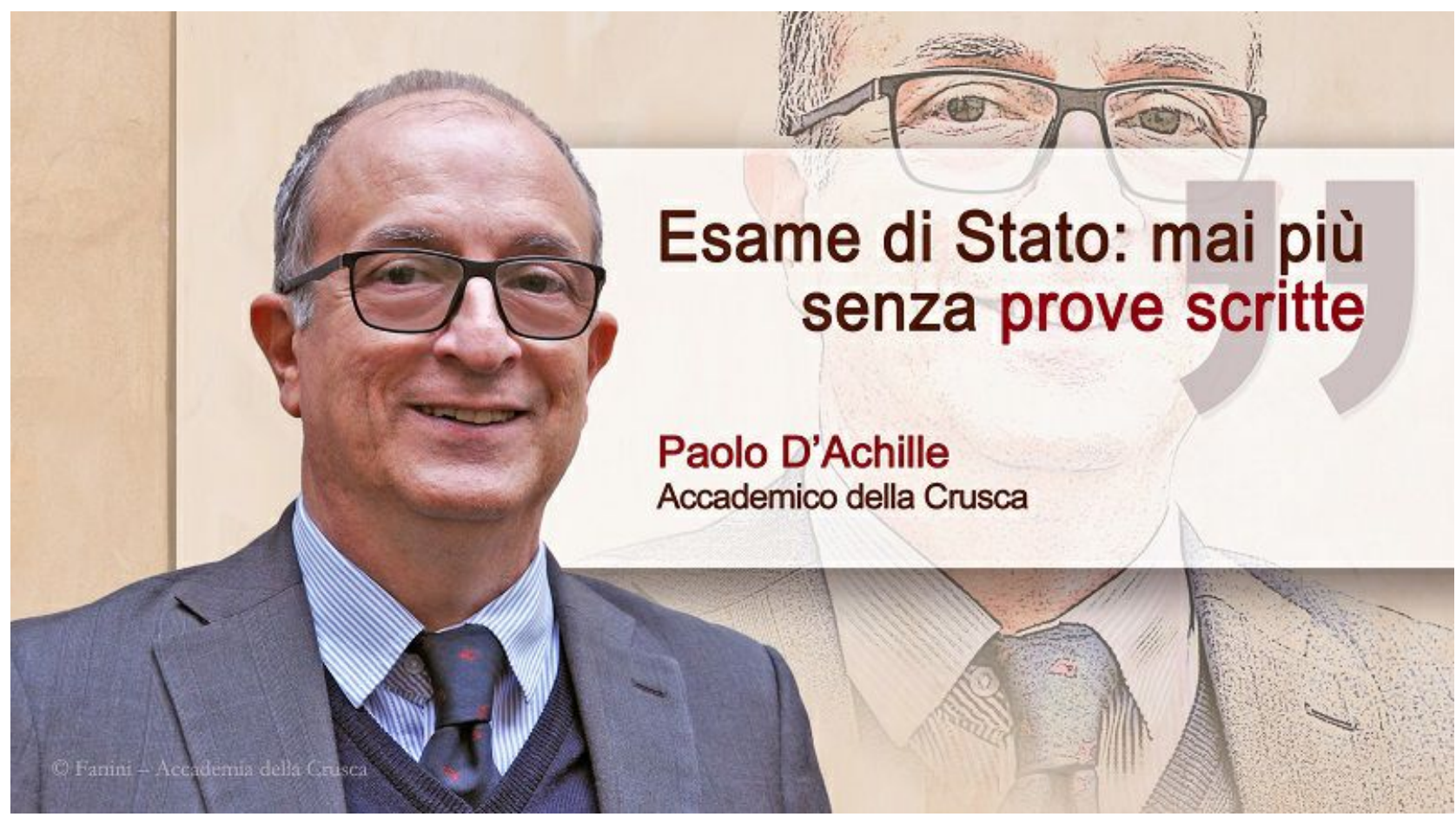

"P riorità". Da quando è scoppiata la pandemia legata al Coronavirus (da un anno e mezzo, all'incirca) sentiamo ripetere quasi ossessivamente questa parola dagli esponenti della politica - ministri dei governi che si sono succeduti alla guida del Paese, parlamentari della maggioranza e dell'opposizione, sindaci e presidenti di regioni, ecc. - a proposito della scuola. Considerata giustamente essenziale per la formazione dei giovanissimi a cui è affidato il nostro futuro, la scuola (parliamo della primaria e della secondaria, lasciando per il momento da parte l'università) è stata (e viene tuttora) indicata come una delle priorità se non addirittura come la priorità. O meglio, la priorità sarebbe il ritorno alla normalità dell'insegnamento in presenza, che la politica si è da tempo impegnata a garantire quanto prima.

Mai come in questo caso le parole sono state finora smentite dai fatti. Lasciamo da parte quello che potremmo definire come il pregresso (continua riduzione dei finanziamenti con conseguente carenza di organici, scarsa attenzione alla formazione e all'aggiornamento dei docenti, sostanziale incuria degli edifici scolastici, ecc.) e concentriamoci sulla situazione contingente, augurandoci che nell'anno scolastico che è appena iniziato o sta per iniziare le cose vadano diversamente. Ebbene, dopo il blocco improvviso della didattica in presenza con la "chiusura totale" del marzo 2020 - a cui si è cercato di ovviare come si poteva, con un repentino passaggio alla didattica a distanza (DAD), alla quale la gran massa dei docenti e dei discenti non era preparata e che non poteva neppure contare su un adeguato supporto tecnologico ed economico, almeno in molte zone d'Italia -, nell'estate 2020 non si tentò neppure di creare le condizioni perché con l'anno scolastico successivo, quello conclusosi circa tre mesi fa, si tornasse alla normalità. Cosi, si sono alternati, senza una vera programmazione, periodi di lezioni in aula (ma spesso a orari ridotti) ad altri di DAD, a volte con la presenza in veste di supplenti (anche per periodi non brevi) di laureati senza esperienza didattica, senza alcuna preparazione specifica sulla 
DAD e senza indicazioni da parte degli istituti scolastici. In questo quadro desolante, sarebbe ingeneroso valutare la (scarsa) preparazione raggiunta nel corso di quest'anno e mezzo dal complesso degli studenti, che ha risentito più che mai degli squilibri socio-economici e culturali di partenza, e sarebbe anche ingiusto addossarne tutte le responsabilità alla DAD in quanto tale, che oggi (diversamente da un anno fa) viene invece quasi unanimemente demonizzata (l'acronimo ufficiale, peraltro, dal settembre 2020 è diventato DDI, didattica digitale integrata).

Non intendo tornare sulle prove INVALSI, argomento di un precedente tema del mese, affrontato da par suo dal collega e amico Rosario Coluccia, anche se ci sarebbe ancora qualcosa da dire, per esempio sul fatto che, col passaggio (dal 2017/20r8) alla somministrazione tramite computer nelle scuole superiori, le domande, prelevate casualmente (in modalità random) da una banca dati preesistente, siano ora secretate, e soprattutto sulla proposta, per la valutazione della comprensione dei testi, di brani letterari ("poco vincolanti", secondo la tipologia di Francesco Sabatini, e dunque aperti in una certa misura allinterpretazione del lettore, per cui il loro significato non è sempre univoco né desumibile da domande a risposta chiusa ed esclusiva). Noteró soltanto che i risultati di queste prove (che documentano comunque, innegabilmente, accentuate carenze di preparazione degli studenti delle superiori in italiano, matematica e inglese), cosi come i rapporti OCSE-PISA (che periodicamente fanno il punto sui livelli di competenze in lettura, matematica e scienze degli studenti di 79 Paesi del mondo e che ci collocano sempre piuttosto in basso nelle classifiche internazionali), non appena vengono pubblicizzati, hanno un'immediata eco sui giornali, alimentano quasi in tempo reale dibattiti e polemiche, ma poi finiscono ben presto con l'essere accantonati ("archiviati", come si dice impropriamente), almeno al di fuori della cerchia degli addetti ai lavori.

E qui si aprirebbe un discorso altrettanto doloroso: ho criticato all'inizio l'atteggiamento dei politici nei confronti della scuola durante la pandemia, ma bisogna ammettere che anche lopinione pubblica, la società civile, la cittadinanza stessa non nutrono per la scuola un particolare interesse, almeno per quello che riguarda il suo ruolo - che in passato è stato invece, giustamente, considerato centrale e che è poi effettivamente venuto meno - di formazione intellettuale delle generazioni più giovani e quindi di ascensore sociale. Per molti genitori, diciamo la verità, la scuola serve soprattutto a "parcheggiare" i figli in un luogo sicuro durante il proprio orario di lavoro, e lo studio a casa non deve togliere troppo tempo allo sport e allo svago: i destini dei figli sembra che si giochino al di fuori dell'istituzione scolastica e una certa attenzione è riservata forse soltanto all'insegnamento/apprendimento dell'inglese, considerato un indispensabile lasciapassare per trovare lavoro (con un occhio soprattutto all'estero).

Ho detto che avrei lasciato da parte l'università, ma anche in questo caso c'è un tema che ricorre periodicamente sulle pagine dei giornali, che vorrei richiamare: l'Italia è stabilmente agli ultimi posti in Europa per quanto riguarda il numero dei laureati. Questo dato, come è noto, non costituisce una novità ed è stato anzi uno degli elementi che hanno portato alla riforma del cosiddetto " $3+2$ ", che, ormai oltre 20 anni fa, ha cambiato radicalmente il sistema universitario italiano. Senza entrare nel merito di quella riforma, possiamo ben dire che, dal punto di vista dell'incremento del numero dei laureati, essa ha risposto solo in parte alle attese. Né hanno migliorato le cose le modifiche successive, culminate con la Legge Gelmini, e gli incessanti interventi del Ministero e dell'ANVUR, che sottopongono ininterrottamente le strutture didattiche degli atenei a continui processi di valutazione, determinando così, in realtà, soltanto o soprattutto una crescita della burocrazia e una progressiva "aziendalizzazione" degli atenei stessi.

A mio parere, c'è un forte rapporto (finora spesso sottovalutato) tra il dato dello scarso numero di laureati (e, più in generale, delle obiettive difficoltà incontrate da moltissimi giovani negli studi 
universitari, conclusi spesso con anni di ritardo) e quello delle carenze disciplinari degli studenti delle scuole superiori che i risultati delle prove INVALSI e dei rapporti PISA-OCSE documentano. Evidentemente le scarse competenze nell'italiano e nella matematica costituiscono un limite un po' per tutti i successivi percorsi di studi universitari, anche quelli che non mettono al centro la nostra lingua - il cui sicuro possesso, accanto a quello di una seconda lingua europea (di fatto l'inglese) è peraltro esplicitamente indicato tra gli obiettivi di tutti i percorsi di laurea triennale - o la scienza dei numeri, forse perché le lacune in questi campi comportano inevitabilmente lacune anche in altri, pur non sottoposti a sondaggi, o forse semplicemente perché le competenze logico-linguistiche sono fondamentali per ogni tipo di studio.

Tutte le riflessioni fatte finora costituiscono solo una premessa alloggetto specifico di questo intervento, che verte sugli esami di maturità, attualmente definiti Esame di Stato, che negli ultimi due anni scolastici si sono svolti con modalità particolari, un po' diverse tra loro, ma sempre con commissioni quasi completamente interne ed esclusivamente attraverso un "colloquio", dunque solo per via orale. C'é poco da recriminare: data la pandemia, è già da considerare un risultato positivo il fatto che, anche sul piano formale, gli esami si siano svolti e che quindi non sia stata data agli studenti la sensazione di diventare todos caballeros senza neppure sostenere la prova. Che poi, di fatto, il numero di coloro che non l'hanno superata sia ancora più esiguo che non in passato, era scontato in partenza, ed è inutile aggiungervi commenti.

C'è stato tuttavia un elemento che ha destato una certa preoccupazione: allindomani della conclusione degli esami, è parso che l'ultima modalità di svolgimento ad alcuni (studenti, docenti, presidi, uffici ministeriali) sia piaciuta, tanto che sono partite un po' in tutta Italia varie iniziative per ostacolarne la possibile istituzionalizzazione. In particolare una docente di materie letterarie, latino e greco di un liceo classico di Pavia, la professoressa Ilaria Rizzini, allarmata, si è fatta promotrice di una petizione per il ripristino delle prove scritte all'Esame di Stato, dato che - come scrive lei stessa - «la misura dell'eliminazione temporanea, indotta dall'emergenza pandemica, rischia di entrare a regime (o, almeno, cosi il Ministro ha lasciato intendere)». Questo, a suo parere, comporterebbe «un ulteriore scadimento della qualità del sistema scolastico nazionale e di rinuncia alla sua funzione formativa nel senso più alto del termine [...]: se davvero l'Esame di Stato (le cui forme sono state molteplici, ma mai prive di prove scritte) sarà decurtato della dissertazione in lingua italiana e di una prova specifica di indirizzo, qualunque essa sia, [...] sarà impossibile impedire che, in breve tempo, simili modalità di accertamento delle conoscenze e delle competenze espressive e argomentative siano messe al bando anche a livello curricolare».

La petizione è arrivata anche all'Accademia della Crusca e io l'ho firmata perché, concordando con quanto affermato dalla professoressa, ho condiviso i suoi timori. Successivamente, però, il 22 luglio scorso il Ministro dell'Istruzione Patrizio Bianchi ha firmato unordinanza che, presentando il calendario scolastico 202I-22, prevede l'inizio dell'Esame di Stato per la scuola secondaria di secondo grado il 22 giugno 2022 con la prima prova scritta d'Italiano, a cui seguiranno altre due prove scritte, a seconda dell'indirizzo, e poi la prova orale finale. Augurandomi, come tutti, che le condizioni generali consentano questo ritorno alla normalità, non mi pare comunque inutile una riflessione sull'importanza della prova scritta.

L'Esame di Stato che si è svolto quest'anno di fatto prevedeva che lo studente o la studentessa mostrasse alla commissione (costituita per la maggior parte dai propri docenti) di saper confezionare bene un pacchetto (ricordo, tra le altre cose, che non è stata valutata direttamente la tesina sulle materie di indirizzo, ma la sua presentazione multimediale) e non la qualità del contenuto all'interno di quel pacchetto (riassorbita di fatto nei punti acquisiti per il "credito scolastico", che hanno 
concorso comunque a determinare il punteggio finale). Che questa modalità diventasse la norma non era davvero ammissibile. L'Esame di Stato non può prescindere dalla verifica del sicuro possesso, da parte degli studenti, della lingua scritta, della loro capacità di argomentare per esporre e sostenere le proprie idee, commentare un brano non conosciuto in precedenza, mostrare doti di analisi e di sintesi nella ricostruzione di un momento storico o di una corrente artistica. La lingua scritta, la cui invenzione - come è stato ormai dimostrato in vari campi di studio - ha rappresentato un momento fondamentale sul piano antropologico, è stata alla base della storia, del progresso scientifico e dello sviluppo della civiltà (e anche il ritardo con cui in Italia si è arrivati all'alfabetizzazione di massa contribuisce a spiegare i deficit scolastici ricordati allinizio). Solo il dominio (sia nella comprensione sia nella produzione) della lingua scritta consente quella capacità di astrazione che è tuttora essenziale per la crescita di ogni singolo individuo. L'esclusione degli scritti dall'Esame di Stato, una volta superata la pandemia, sarebbe (prudenzialmente, preferisco usare ancora il condizionale presente) davvero un'assurdità, tanto più perché, come scrive la professoressa Rizzini, ciò determinerebbe inevitabilmente il progressivo disinteresse per le prove scritte nella didattica curriculare (come del resto è in parte già avvenuto, per motivi di forza maggiore, durante la pandemia) e, conseguentemente, un ulteriore peggioramento del livello di preparazione degli studenti non solo in italiano, ma anche nelle lingue classiche (visto che la pratica della traduzione verrebbe largamente disattesa, come lo è stata negli scorsi mesi), nella matematica, in tutte le discipline che caratterizzano i vari indirizzi di studio per le quali il vecchio esame di maturità prevedeva una prova scritta. Invece, incentivare a scuola la produzione scritta potrebbe persino favorire la ripresa dell'ascensore sociale oggi fermo. È anche da notare che nel modello scolastico del mondo anglosassone l'orale ha scarsissimo peso, perché le prove sono tutte scritte; ma, stranamente, noi non teniamo assolutamente conto dell'importanza della scrittura nella "vincente" cultura anglosassone, che pure qui in Italia costituisce un punto di riferimento, da cui attingiamo continuamente metodi e pratiche.

Vorrei aggiungere ancora qualcosa per quanto riguarda specificamente la lingua e letteratura italiana: privata di una prova scritta specifica (e anche del suo uso nella traduzione da un'altra lingua, antica o moderna), è stata rappresentata disciplinarmente (e dunque valutata) all'interno dell'Esame di Stato di quest'anno solo dall'analisi di un brano letterario incluso nel programma svolto. Ma, date anche le modalità della didattica ricordate all'inizio, spesso i programmi (di tutte le materie invero, non solo di italiano) sono stati forzatamente decurtati e ben pochi autori della nostra tradizione letteraria sono stati letti e commentati direttamente. Figuriamoci quindi quale peso effettivo possa aver avuto questa prova! Tra l'altro, so per certo che alcuni programmi di italiano hanno presentato la lettura di due soli canti del Paradiso (proprio nell'anno dantesco, celebrato da moltissime manifestazioni!) e ho motivo di credere che in ben pochi casi sia stato chiesto ai candidati di commentare un passo di Dante. Ma rileverei piuttosto (perché la cosa non riguarda solo la situazione emergenziale) come da vari anni le indicazioni ministeriali (di programmi veri e propri non si parla più) e i libri di testo prevedano che nell'ultimo anno si studino gli autori contemporanei (dalla metà dell'Ottocento a tutto il Novecento). Queste indicazioni vengono però spesso largamente disattese. Posso segnalare al riguardo che la collega Simona Costa, che fino allo scorso anno accademico ha insegnato Letteratura italiana contemporanea nel mio stesso ateneo di Roma Tre, già qualche anno fa pretese, giustamente, che nei curricula di Italianistica della laurea triennale in Lettere fosse inserito un modulo istituzionale di Letteratura italiana contemporanea, obbligatorio e propedeutico a un secondo modulo monografico, perché molte matricole non sapevano neppure chi fosse Montale (che era, insieme a Moravia, l'ultimo autore inserito nel "mio" programma di italiano, quando affrontai l'esame di maturità, ormai quasi cinquant'anni fa!). Forse pure sul rispetto delle indicazioni ministeriali qualche verifica andrà fatta, quando si tornerà alla normalità, valutando anche la concreta possibilità della loro applicazione in rapporto alle ore di insegnamento effettivo. 
Concludo dicendo che so benissimo che, all'interno del variegato mondo della scuola italiana, ci sono situazioni in cui l'impegno congiunto (ciascuno per la sua parte) di docenti e studenti, ma anche di presidi, non docenti e genitori, riesce sempre e comunque a ottenere risultati più che soddisfacenti e a volte addirittura straordinari, e sono certo che questo è avvenuto anche nel corso della pandemia. Ma a mio parere le punte di eccellenza esistenti, che sono certamente da segnalare e da valorizzare, non devono far dimenticare che la situazione generale della scuola è alquanto diversa. A questa realtà soprattutto bisogna guardare, per cercare di migliorarla e per evitare l'insorgere di ulteriori criticità.

\section{Cita come:}

Paolo D'Achille, Esame di Stato: mai più senza prove scritte , "Italiano digitale", XVIII, 2021/3 (lugliosettembre)

DOI: $10.35948 / 2532-9006 / 2021.10601$

Copyright 2021 Accademia della Crusca

Pubblicato con licenza creative commons CC BY-NC-ND 